\title{
Regorafenib combined with transarterial chemoembolization for unresectable hepatocellular carcinoma: a real-world study
}

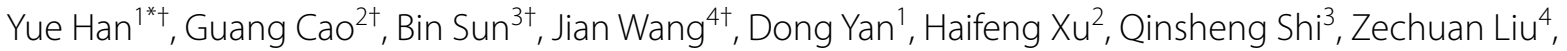
Weihua Zhi ${ }^{1}$, Liang $\mathrm{Xu}^{2}$, Bojun Liu ${ }^{3}$ and Yinghua Zou ${ }^{4}$

\begin{abstract}
Background: The benefits and tolerability of transarterial chemoembolization (TACE) combined with regorafenib as a second-line therapy has not been reported for unresectable hepatocellular carcinoma (HCC). This study aimed to explore the benefits and tolerability of TACE combined with second-line regorafenib in patients with unresectable advanced HCC and failure to first-line treatment.

Methods: This was a multicenter retrospective study of patients with progression after first-line sorafenib and/or lenvatinib between 01/2019 and 04/2020 at four tertiary hospitals in China. The patients were treated with TACE. Then, 5-7 days after the first TACE, the patients started taking regorafenib for 3 weeks every 4-week cycle. The overall survival (OS), time to progression (TTP), progression-free survival (PFS), and adverse events (AEs) were observed.

Results: The median follow-up was 5.6 (range $0.7,17.0$ ) months. The median age was 60 (range 35, 79) years. There were 32 (84.2\%) males. The patients underwent a median of three TACE sessions (range 1-13). The initial doses of regorafenib were $20 \mathrm{mg} / \mathrm{d}(n=1,2.6 \%), 80 \mathrm{mg} / \mathrm{d}(n=10,26.3 \%), 120 \mathrm{mg} / \mathrm{d}(n=15,39.5 \%)$, and $160 \mathrm{mg} / \mathrm{d}(n=11$, 28.9\%). The incidence of grade $3 / 4$ AEs was $15.8 \%$. Two patients stopped regorafenib due to AEs. The median OS was 14.3 months. The median PFS and TTP were $9.1(95 \% \mathrm{Cl}$ 4.0, 14.2) and $9.1(95 \% \mathrm{Cl} 5.5,12.8)$ months, respectively.

Conclusions: The present study provides real-world evidence indicating that regorafenib combined with TACE was beneficial and tolerable in patients with unresectable HCC. Additional prospective large-scale studies are required for confirmation.
\end{abstract}

Keywords: Transarterial chemoembolization, Unresectable, Hepatocellular carcinoma, Regorafenib, Survival

*Correspondence: doctorhan@163.com

†Yue Han, Guang Cao, Bin Sun and Jian Wang have contributed equally to this work

${ }^{1}$ Department of Interventional Therapy, National Cancer Center, National Clinical Research Center for Cancer, Cancer Hospital, Chinese Academy of Medical Sciences and Peking Union Medical College, Beijing 100021, China

Full list of author information is available at the end of the article

\section{Background}

Hepatocellular carcinoma (HCC) is a highly lethal invasive carcinoma arising in the liver and accounts for $75-85 \%$ of all liver cancers [1-4]. The worldwide agestandardized annual mortality rates of liver cancer are 13.9 per 100,000 in men and 4.9 per 100,000 in women [3]. The most important risk factors for HCC are infection with hepatitis $\mathrm{B}$ or hepatitis $\mathrm{C}$ and/or preexisting liver cirrhosis, and the incidence of HCC generally follows the geographical distribution of hepatitis $\mathrm{B}$ and $C$ viruses $[1,5,6]$. In China, liver cancer ranks fourth 
among the most common malignant tumors and the second among the causes of cancer death $[7,8]$. Due to the insidious onset of liver cancer, the symptoms are not obvious or typical, and most patients are diagnosed with advanced disease $[1,5,6,9,10]$. In addition, patients with advanced-stage HCC often cannot undergo radical treatment (surgical excision or liver transplantation) due to the status of liver function, shortage of donor liver source, and metastasis $[1,4,6,11-13]$.

Transarterial chemoembolization (TACE) and/or systemic drugs (such as sorafenib as first-line treatment) are recommended to treat inoperable patients with advanced HCC $[1,4,6,12]$. Nevertheless, the efficacy of TACE alone is relatively poor, with complete response rates of only $0-4.8 \%$ [14]. The objective response rate (ORR) of TACE alone is lower than TACE combined with systemic treatment [15], and tumors still progress even after multiple TACE. Nevertheless, the efficacy of systemic treatment alone is also relatively poor [16-18]. Sorafenib, combined with TACE, has been proven to yield good outcomes [19]. Many studies confirmed that the efficacy of sorafenib combined with TACE in the treatment of advanced HCC is better than that of either TACE and antiangiogenic treatments alone, with an acceptable safety profile $[15,19-21]$.

Regorafenib (Bay 73-4506) is an oral multikinase inhibitor whose antitumor effect is achieved by blocking and inhibiting the activity of multiple protein kinases involved in tumor angiogenesis, tumorigenesis, metastasis, and tumor immunity $[22,23]$. The international, multicenter, placebo-controlled phase III RESORCE trial confirmed that compared with placebo, patients with advanced HCC receiving regorafenib treatment had higher ORR and disease control rate (DCR) and longer progressionfree survival (PFS), time to progression (TTP), and overall survival (OS) [24]. Therefore, regorafenib has become a standard second-line treatment for HCC after sorafenib treatment.

Since many studies confirmed the efficacy of sorafenib combined with TACE $[15,19-21]$, we hypothesized that second-line regorafenib combined with TACE might have better efficacy in patients with HCC who failed to undergo first-line TACE with or without sorafenib or rivatinib. Therefore, this multicenter retrospective study was conducted to explore the benefits and tolerability of TACE combined with second-line regorafenib in patients with unresectable advanced HCC and failure to first-line treatment.

\section{Methods}

\section{Patients}

This multicenter retrospective study included patients with progression after first-line sorafenib and/or lenvatinib between January 2019 and April 2020 at four tertiary hospitals in China. All experiments were performed in accordance with the Declaration of Helsinki. This study was approved by Chinese Ethics Committee of Registering Clinical Trials (ChiECRCT20210138). The requirement for individual informed consent was waived by Chinese Ethics Committee of Registering Clinical Trials.

The inclusion criteria were: $(1)>18$ years of age; (2) pathologically or clinically diagnosed HCC[25]; (3) unresectable or refused surgery; (4) first-line sorafenib and/ or lenvatinib treatment; (5) Eastern Cooperative Oncology Group (ECOG) performance status (PS) score of 0-2; (6) Barcelona clinic liver cancer (BCLC) stage B-C; (7) Child-Pugh grade A or B; (8) TACE; and (9) underwent TACE combined with regorafenib. The exclusion criteria were: (1) incomplete data; (2) complicated with other serious diseases, including severe cardiovascular and cerebrovascular diseases, severe liver function injury (2 times higher than reference value), severe renal injury (eGFR $<45 \mathrm{ml} / \mathrm{min} / 1.73 \mathrm{~m}^{2}$ ), and hematologic disease; (3) history of other malignant tumors; (4) pregnant or lactating women; or (5) participation in a clinical trial.

\section{Treatment}

The patients were treated with TACE. Then, 5-7 days after the first TACE, the patients started taking regorafenib for 3 weeks every 4-week cycle.

\section{Data collection and definitions}

All data were collected from the electronic health records, including age, sex, body mass index (BMI), complications, pathological diagnosis ratio, liver cirrhosis, child-Pugh, ECOG score, BCLC stage, macrovascular invasion, extrahepatic spread, $\alpha$-fetoprotein (AFP), hepatitis $\mathrm{B} / \mathrm{C}$ infection, number of intrahepatic lesions, maximum lesion diameter, and previous treatments. The treatment-related variables were treatment time, initial dosage, dosage adjustment, course of treatment, reasons for withdrawal, time, method, frequency of TACE treatment, and reasons for termination of treatment.

The primary outcomes were PFS, TTP, and OS, calculated from the first TACE treatment. The secondary outcomes were complete response (CR), partial response $(\mathrm{PR})$, progressive disease $(\mathrm{PD})$, stable disease $(\mathrm{SD})$, and ORR (according to mRECIST), drug safety (CTCAE 4.03), and surgical complications (Clavien classification). The last follow-up was on June 15, 2020.

\section{Statistical analysis}

The continuous variables with a normal distribution were expressed as means \pm standard deviations, and those with a skewed distribution were expressed as medians 
(ranges). Categorical variables were expressed as n (\%). Survival analysis was performed using the Kaplan-Meier method and the log-rank test. Univariable and multivariable Cox proportional hazards models were used to analyze the prognostic factors. The variables with P-values $<0.05$ in the univariable analyses were included in the multivariable analysis using the forward method. Two-sided P-values $<0.05$ were considered statistically significant.

\section{Results}

\section{Characteristics of the patients}

A total of 38 patients were included in the study. The median follow-up was 5.6 (range $0.7,17.0$ ) months. The median age was 60 (range 35,79$)$ years. There were 32 $(84.2 \%)$ males. Eighteen (47.4\%) patients were in BCLC stage B and $20(52.6 \%)$ were in BCLC stage C. ECOG performance status was 0 in $20(52.6 \%)$ patients, 1 in 13 (34.2\%), and 2 in five (13.2\%), respectively. Regarding the Child-Pugh stage, one (2.6\%) patient was grade B, and the others were grade A. There were $12(31.6 \%)$ patients with macrovascular invasion and $13(34.2 \%)$ with extrahepatic metastases. Previous treatment included surgery in $13(34.2 \%)$ patients, TACE in $36(94.7 \%)$, and radiation therapy in six (15.8\%). Sorafenib was the main drug for previous systemic treatment, which was used in 33 (86.8\%) patients (Table 1).

\section{Treatment profile of TACE combined with regorafenib}

Patients were treated with cTACE $(\mathrm{n}=20,52.6 \%)$ or D-TACE $(n=17,44.7 \%)$, for a median of 3 (range 1,13 ) sessions. The initial doses of regorafenib were $20 \mathrm{mg} / \mathrm{d}$ $(\mathrm{n}=1,2.6 \%), 80 \mathrm{mg} / \mathrm{d}(\mathrm{n}=10,26.3 \%), 120 \mathrm{mg} / \mathrm{d}(\mathrm{n}=15$, $39.5 \%)$, and $160 \mathrm{mg} / \mathrm{d}(\mathrm{n}=11,28.9 \%)$. During treatment, 15 patients stopped regorafenib because of progression $(\mathrm{n}=9,60.0 \%)$, intolerance $(\mathrm{n}=5,33.3 \%)$, and others $(\mathrm{n}=1,6.1 \%)$ (Table 2).

\section{Outcomes}

At the last follow-up, 15 patients experienced a progression, and seven patients had died. The best treatment responses were $\mathrm{CR}$ in one patient (2.6\%), $\mathrm{PR}$ in two (5.3\%), SD in 26 (68.4\%), and PD in nine (23.7\%), for an ORR of $7.9 \%$ and a DCR of $76.3 \%$ (Table 3). The median PFS was 9.1 (range: 4.0, 14.2) months, the median TTP was 9.1 (range $5.5,12.7$ ) months, and the median OS was 14.3 (NA, NA) months (Fig. 1).

\section{Univariable and multivariable analyses}

Cox univariable analyses were performed with PFS as the outcome. AFP $\geq 400 \mathrm{ng} / \mathrm{ml}$ was associated with lower PFS than AFP $<400 \mathrm{ng} / \mathrm{ml}$ (log-rank $\mathrm{P}=0.025$; $\mathrm{HR}=3.046,95 \%$ CI 1.092-8.497, $\mathrm{P}=0.033)($ Fig. $2 \mathrm{~A}$ and
Table 1 Characteristics of the patients

\begin{tabular}{|c|c|}
\hline Characteristic & Total $(n=38)$ \\
\hline Age (years) & $59.4 \pm 9.2$ \\
\hline Median (range) & $60(35,79)$ \\
\hline \multicolumn{2}{|l|}{$\operatorname{Sex}, n(\%)$} \\
\hline Male & $32(84.2)$ \\
\hline Female & $6(15.8)$ \\
\hline \multicolumn{2}{|l|}{ ECOG performance status, $n$ (\%) } \\
\hline 0 & $20(52.6)$ \\
\hline 1 & $13(34.2)$ \\
\hline 2 & $5(13.2)$ \\
\hline \multicolumn{2}{|l|}{$B C L C$ grade, $n(\%)$} \\
\hline B & $18(47.4)$ \\
\hline C & $20(52.6)$ \\
\hline \multicolumn{2}{|l|}{ Hepatitis, $n(\%)$} \\
\hline Hepatitis B & $32(84.2)$ \\
\hline Hepatitis C & $2(5.3)$ \\
\hline No & $4(10.5)$ \\
\hline Maximum tumor size (cm) & $3.75(0.9,15.1)$ \\
\hline \multicolumn{2}{|l|}{ Tumor number, $n(\%)$} \\
\hline Single & $4(10.5)$ \\
\hline Multiple & $32(84.2)$ \\
\hline Unknown & $2(5.3)$ \\
\hline \multicolumn{2}{|l|}{ Child-Pugh grade, $n(\%)$} \\
\hline A & $37(97.4)$ \\
\hline B & $1(2.6)$ \\
\hline Macrovascular invasion, n (\%) & $12(31.6)$ \\
\hline $\mathrm{AFP} \geq 400 \mu \mathrm{g} / \mathrm{L}, \mathrm{n}(\%)$ & $11(28.9)$ \\
\hline \multicolumn{2}{|l|}{ Comorbidity, n (\%) } \\
\hline Hypertension & $15(39.5)$ \\
\hline Diabetes Mellitus & $4(10.5)$ \\
\hline Extrahepatic metastasis, n (\%) & $13(34.2)$ \\
\hline \multicolumn{2}{|l|}{ Prior local therapy, $n$ (\%) } \\
\hline Surgery & $13(34.2)$ \\
\hline Ablation & $20(52.6)$ \\
\hline TACE & $36(94.7)$ \\
\hline Radiotherapy & $6(15.8)$ \\
\hline \multicolumn{2}{|l|}{ Prior systemic therapy, n (\%) } \\
\hline Sorafenib & $33(86.8)$ \\
\hline Lenvatinib & $1(2.6)$ \\
\hline Lenvatinib sequential to sorafenib & $4(10.5)$ \\
\hline Follow-up (months), median (range) & $5.6(0.7,17.0)$ \\
\hline
\end{tabular}

ECOG Eastern Cooperative Oncology Group, BCLC Barcelona clinic liver cancer, AFP a-fetoprotein, TACE transarterial chemoembolization

Additional file 1: Table S1). Tumor size $>3.75 \mathrm{~cm}$ was associated with PFS (log-rank $\mathrm{P}=0.04 ; \mathrm{HR}=2.822$, 95\% CI 1.011-7.879, $\mathrm{P}=0.048$ ) (Fig. $2 \mathrm{~B}$ and Additional file 1: Table S1). Initial dose of Regorafenib at 120 or $160 \mathrm{mg} / \mathrm{d}$ was associated with higher PFS than at $80 \mathrm{mg} / \mathrm{d}$ (log-rank $\mathrm{P}=0.002 ; \mathrm{HR}=0.209,95 \%$ CI $0.07-0.623, \mathrm{P}=0.005$ ) 
Table 2 Treatment profile

\begin{tabular}{lc}
\hline Variable & Total $(\mathbf{n}=\mathbf{3 8})$ \\
\hline TACE method, $n$ (\%) & \\
cTACE & $20(52.6 \%)$ \\
D-TACE & $17(44.7 \%)$ \\
Unknown & $1(2.6 \%)$ \\
TACE sessions, n (range) & $3(1,13)$ \\
Regorafenib initial dosage, $n(\%)$ & \\
20 mg/d & $1(2.6 \%)$ \\
80 mg/d & $10(26.3 \%)$ \\
120 mg/d & $15(39.5 \%)$ \\
160 mg/d & $11(28.9 \%)$ \\
Unknown & $1(2.6 \%)$ \\
Stopped regorafenib & $15(39.5 \%)$ \\
Reasons for stopping & \\
Progression & $9(23.7 \%)$ \\
Intolerance & $5(13.2 \%)$ \\
Other & $1(2.6 \%)$ \\
\hline ECOG Eastern Cooperative Oncology Group
\end{tabular}

ECOG Eastern Cooperative Oncology Group

Table 3 Tumor response

\begin{tabular}{lc}
\hline Variable & Total $(\mathbf{n}=\mathbf{3 8})$ \\
\hline Best response, $n(\%)$ & \\
Complete response & $1(2.6)$ \\
Partial response & $2(5.3)$ \\
Stable disease & $26(68.4)$ \\
Progressive disease & $9(23.7)$ \\
Objective response rate, $\mathrm{n}(\%)$ & $3(7.9)$ \\
Disease control rate, $\mathrm{n}(\%)$ & $29(76.3)$ \\
6-month PFS rate, $\%(95 \% \mathrm{Cl})$ & $59.8(41.8,77.8)$ \\
\hline
\end{tabular}

(Fig. 2C and Additional file 1: Table S1). Patients achieving $\mathrm{CR}, \mathrm{PR}$, or SD had a better PFS than those with PD (log-rank $\mathrm{P}<0.001 ; \mathrm{HR}=5.998,95 \%$ CI 2.274-15.820, $\mathrm{P}<0.001$ ) (Fig. 2D and Additional file 1: Table S1).

After adjusting for AFP levels and tumor size in the Cox multivariable model, the initial dosage of 120 or $160 \mathrm{mg} / \mathrm{d}(\mathrm{HR}=0.216,95 \% \mathrm{CI} 0.061-0.765, \mathrm{P}=0.018)$ and patients achieving $\mathrm{PD}(\mathrm{HR}=5.607,95 \% \mathrm{CI} 1.896-$ $16.578, \mathrm{P}=0.002)$ were independently associated with PFS (Additional file 1: Table S1).

Cox univariable analyses were performed with TTP as the outcome. AFP $\geq 400 \mathrm{ng} / \mathrm{ml}$ was associated with shorter TTP than AFP $<400 \mathrm{ng} / \mathrm{ml}$ (log-rank $\mathrm{P}=0.006$; $\mathrm{HR}=4.19$, 95\% CI 1.391-12.596, $\mathrm{P}=0.011$ ) (Fig. 3A and Additional file 1: Table S2). Maximum tumor size $>3.75 \mathrm{~cm}$ was associated with shorter TTP (log-rank $\mathrm{P}=0.029 ; \mathrm{HR}=3.252$, 95\% CI 1.077-9.821, $\mathrm{P}=0.037$ ) (Fig. 3B and Additional file 1: Table S2). Regorafenib at 120 or $160 \mathrm{mg} / \mathrm{d}$ was associated with longer TTP than at $80 \mathrm{mg} / \mathrm{d}$ (log-rank $\mathrm{P}=0.039 ; \mathrm{HR}=0.293$, 95\% CI $0.085-$ 1.006, $\mathrm{P}=0.051$ ) (Fig. $3 \mathrm{C}$ and Additional file 1: Table S2). Patients achieving CR, PR, or SD had a longer TTP than those with PD (log-rank $\mathrm{P}=0.001 ; \mathrm{HR}=4.858,95 \% \mathrm{CI}$ 1.731-13.635, $\mathrm{P}=0.003$ ) (Fig. $3 \mathrm{D}$ and Additional file 1: Table S2).

After adjusting for tumor size in the Cox multivariable model, a maximum tumor size $>3.75 \mathrm{~cm} \quad(\mathrm{HR}=5.544$, 95\% CI 1.616-19.02, $\mathrm{P}=0.006)$ and patients achieving $\mathrm{PD}(\mathrm{HR}=7.691,95 \%$ CI $2.37-24.964, \mathrm{P}=0.001)$ were independently associated with TTP (Additional file 1: Table S2).

Finally, Cox univariable analyses were performed with OS as the outcome. AFP was not associated with OS (log-rank $\mathrm{P}=0.057 ; \mathrm{HR}=3.487$, 95\% CI 0.857-14.196, $\mathrm{P}=0.081$ ) (Fig. $4 \mathrm{~A}$ and Additional file 1: Table S3). Tumor size was not associated with OS (log-rank $\mathrm{P}=0.648 ; \quad \mathrm{HR}=1.37, \quad 95 \% \quad \mathrm{CI} \quad 0.338-5.55, \quad \mathrm{P}=0.66$ )

(Fig. 4B and Additional file 1: Table S3). Regorafenib at
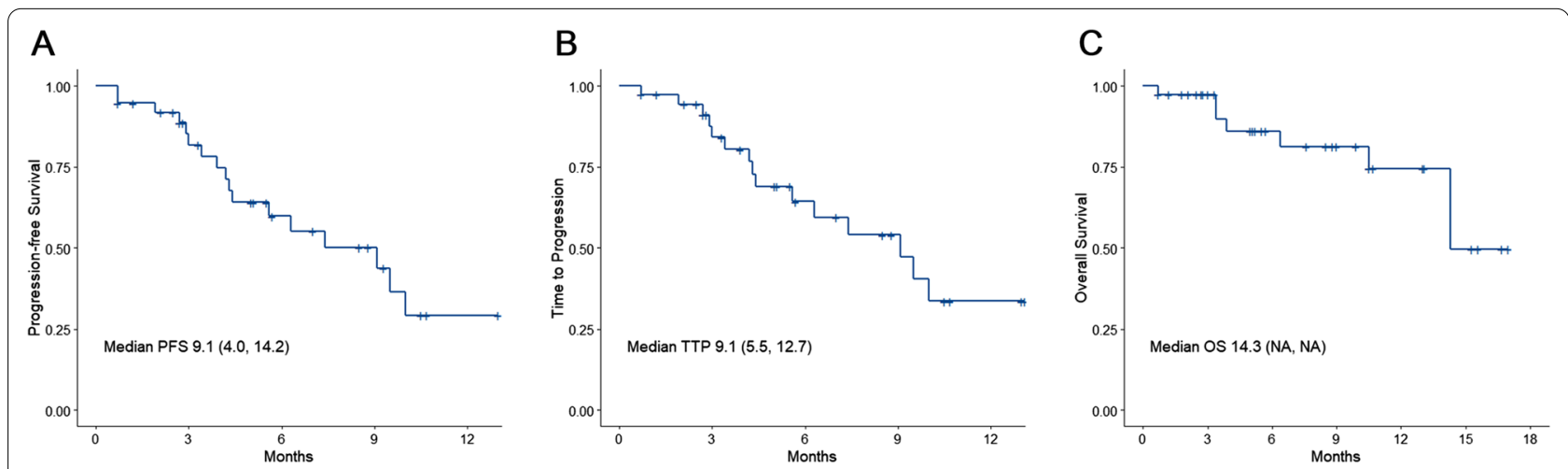

Fig. 1 Kaplan-Meier plots of median A progression-free survival (PFS), B time-to-progression (TTP), and C overall survival (OS) in patients treated with TACE plus regorafenib 


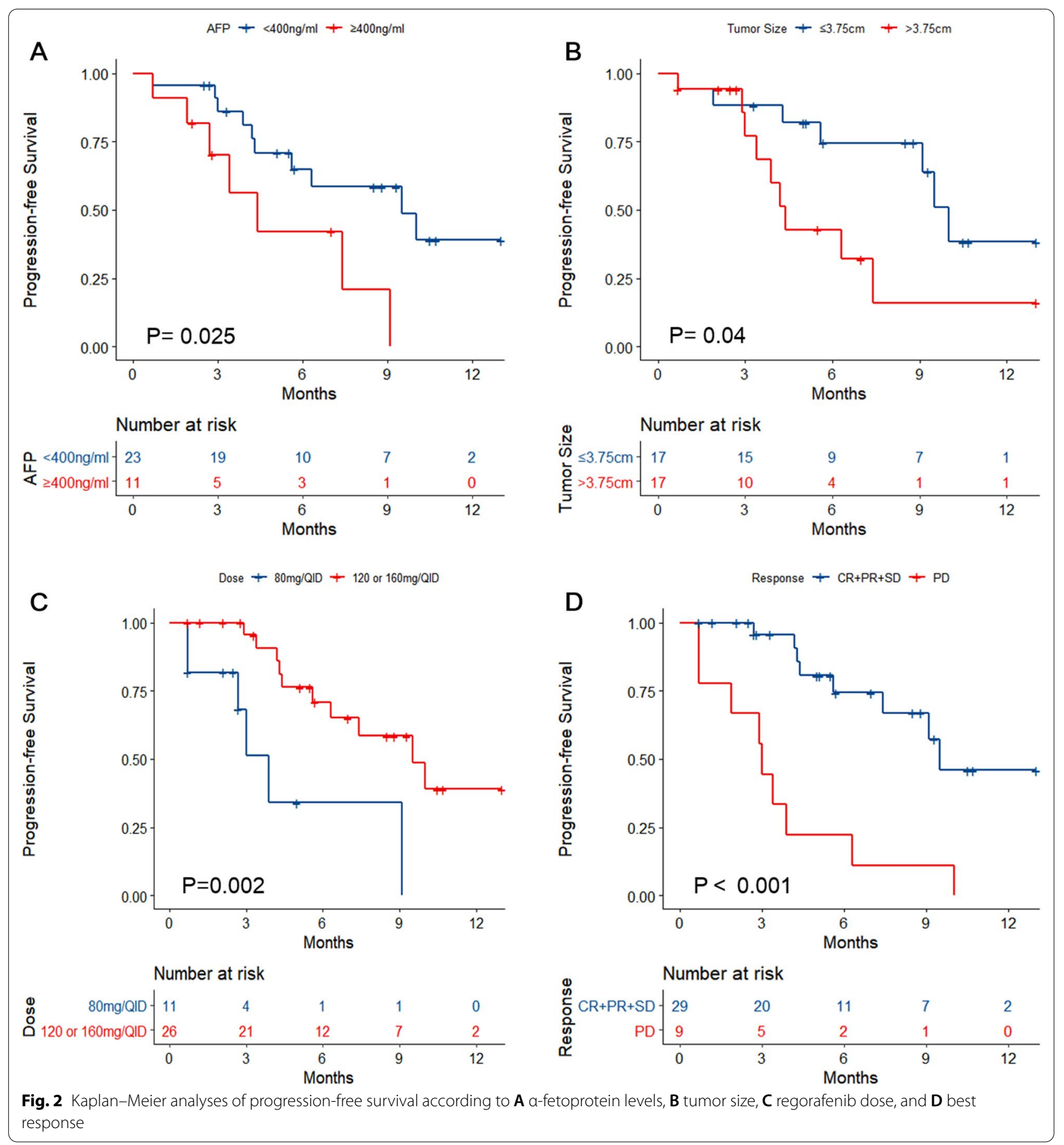

120 or $160 \mathrm{mg} / \mathrm{d}$ was associated with longer OS than at $80 \mathrm{mg} / \mathrm{d}$ (log-rank $\mathrm{P}<0.001 ; \mathrm{HR}=0.043$, 95\% CI 0.005$0.397, \mathrm{P}=0.006$ ) (Fig. 4C and Additional file 1: Table S3). Patients achieving CR, PR, or SD had a longer OS than those with PD (log-rank $\mathrm{P}=0.001$; $\mathrm{HR}=9.151,95 \% \mathrm{CI}$ 1.823-45.939, $\mathrm{P}=0.007$ ) (Fig. $4 \mathrm{D}$ and Additional file 1 : Table S3).
In the Cox multivariable model, an initial dosage at 120 or $160 \mathrm{mg} / \mathrm{d}(\mathrm{HR}=0.049,95 \%$ CI $0.004-0.535$, $\mathrm{P}=0.013)$ and patients achieving $\mathrm{PD}(\mathrm{HR}=6.497,95 \%$ CI $1.103-38.284, \mathrm{P}=0.039)$ were independently associated with OS (Additional file 1: Table S3). 


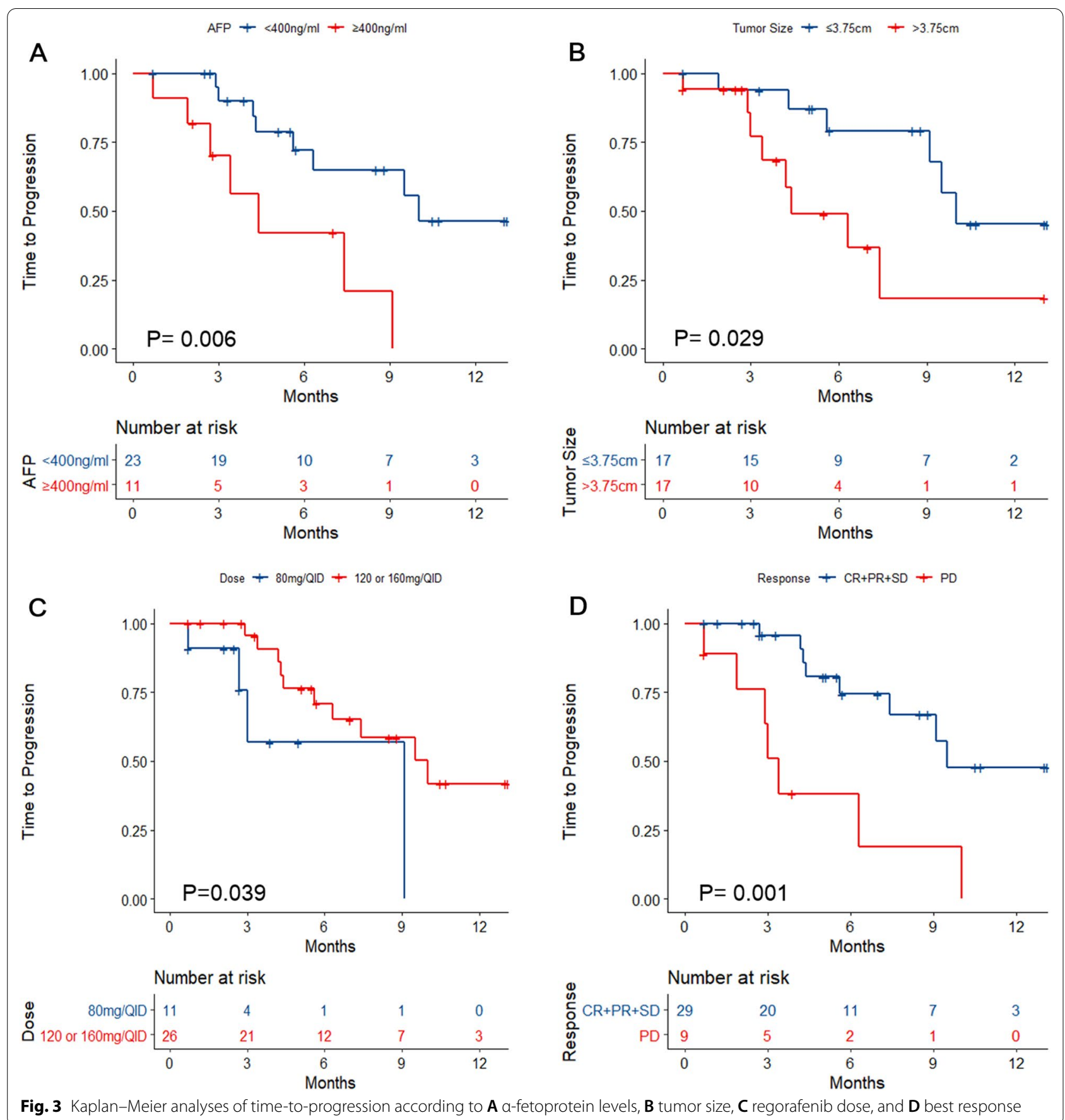

\section{Toxicity}

As shown in Table 4, no grade 5 AEs (death) were observed. After TACE, the complications included nausea $(n=11,28.9 \%)$, pain $(n=11,28.9 \%)$, vomiting $(n=3$, $7.9 \%)$, and fever $(\mathrm{n}=4,10.5 \%)$. Seven $(18.4 \%)$ patients experienced at least one grade 3-4 AE. There were two cases of drug withdrawal due to AEs. The most common AEs ( $\geq 10 \%$ occurrence) were hand-foot syndrome $(n=8$,
$21.1 \%)$, anemia $(\mathrm{n}=4,10.5 \%)$, leukopenia $(\mathrm{n}=4,10.5 \%)$, thrombocytopenia $(n=5,13.2 \%)$, and elevated aspartate transaminase levels $(\mathrm{n}=4,10.5 \%)$.

\section{Discussion}

To the best of our knowledge, this study is the first to explore the feasibility, prognosis, and toxicity of TACE combined with regorafenib to treat patients with 


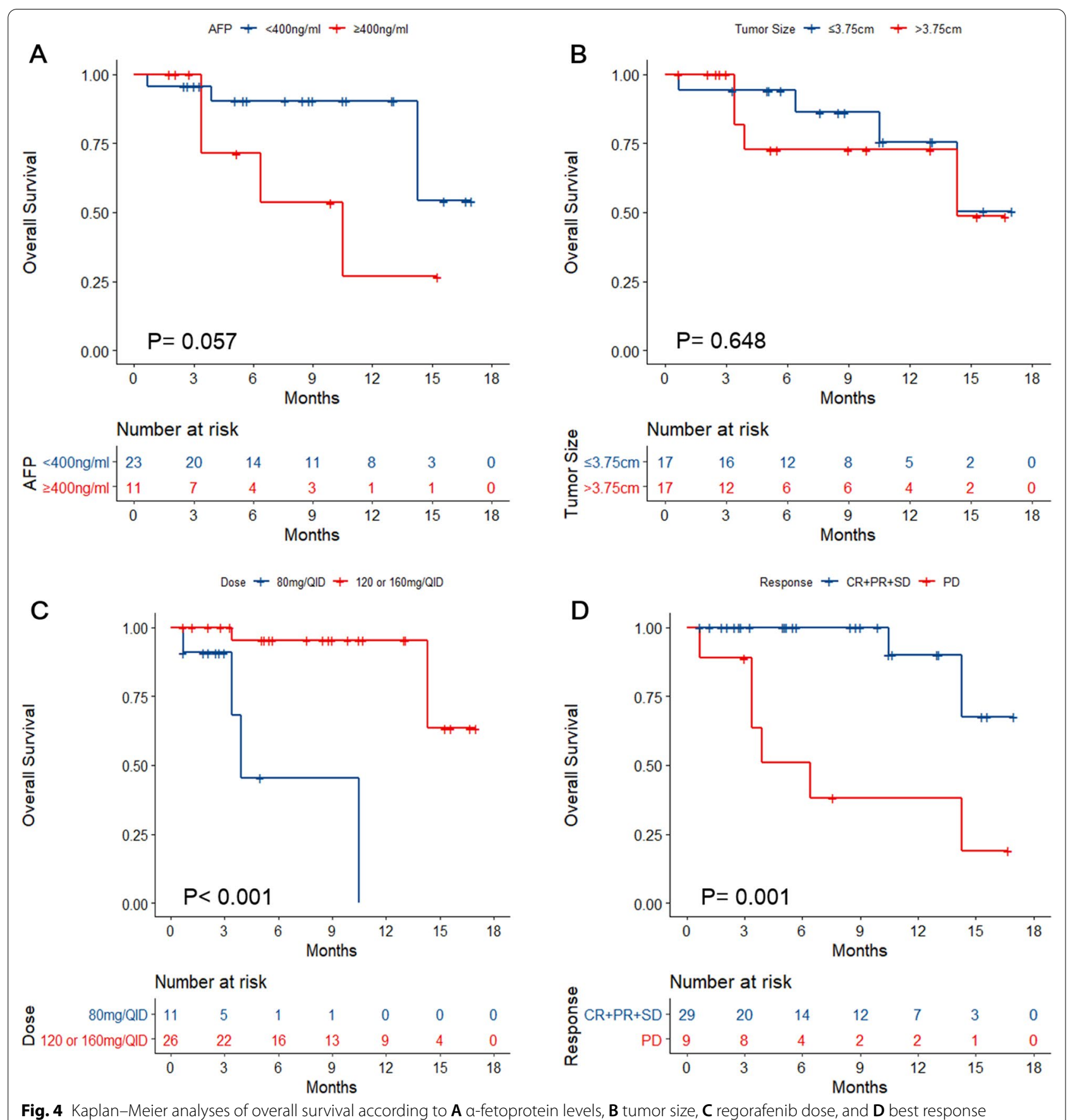

unresectable HCC and failure to first-line treatment. The results showed promising DCR, 6-month PFS rate, and median OS, and the treatment was tolerable. Patients who achieved disease control after TACE combined with regorafenib might have a better prognosis. TACE combined with regorafenib might be an alternative treatment for unresectable HCC after failure to first-line treatment.
The patients included in this study were those who received first-line targeted therapy for unresectable HCC. Of them, most were treated with TACE (94.7\%), followed by ablation (52.6\%) and surgery (34.2\%). The median number of TACE sessions was $3(1,13)$. Among the patients, 14 received sorafenib, five received ranvatinib, and four received both. Higher numbers of early treatments might increase the risk of adverse reactions. 
Table 4 Postoperative complications and adverse events

\begin{tabular}{ll}
\hline & Total $(\mathbf{n}=\mathbf{3 8})$ \\
\hline Postoperative complications & \\
Nausea & $11(28.9 \%)$ \\
Pain & $11(28.9 \%)$ \\
Vomiting & $3(7.9 \%)$ \\
Fever & $4(10.5 \%)$ \\
Adverse events & \\
Grade 3-4 adverse events & $7(18.4 \%)$ \\
Hand-foot syndrome & $8(21.1 \%)$ \\
Anemia & $4(10.5 \%)$ \\
Leukopenia & $4(10.5 \%)$ \\
Thrombocytopenia & $5(13.2 \%)$ \\
Elevated aspartate transaminase & $4(10.5 \%)$ \\
Hyperbilirubinemia & $2(5.3 \%)$ \\
Diarrhea & $4(10.5 \%)$ \\
Nausea & $1(2.6 \%)$ \\
Hypertension & $2(5.3 \%)$ \\
Rash & $2(5.3 \%)$ \\
\hline
\end{tabular}

Nevertheless, in this study, $13.2 \%$ of the patients had drug withdrawal due to intolerance, which was lower than the $25 \%$ observed in the RESORCE study [24]. In addition, flexible treatment, such as lower initial dosage and ondemand TACE, might improve tolerance and should be explored.

In this study, TACE combined with regorafenib was used as a second-line treatment, leading to a median PFS of 9.1 (4.0, 14.2) months, median TTP of 9.1 (5.5, 12.7) months, and median OS of 14.3 (NA, NA) months. In the RESORCE study, regorafenib monotherapy was used in advanced HCC patients after sorafenib, with a median PFS of 3.1 months (95\% CI 2.8-4.2), median TTP of 3.2 months (95\% CI 2.9-4.2), and a median OS of 10.6 months (95\% CI 9.1-12.1) [24]. Lee et al. [26] used regorafenib monotherapy after progression to sorafenib, and the median PFS was 2.7 months (95\% CI 2.5-2.9 months), the median TTP was 2.6 months (95\% CI 2.4-2.8 months), and the median OS was 10.0 months (95\% CI 8.4-11.6 months) [26]. Compared with monotherapy, TACE combined with systemic therapy (using regorafenib after sorafenib treatment) might lead to longer PFS, TTP, and OS, but this will have to be confirmed.

The DCR in this study was $76.3 \%$, higher than in the RESORCE study (65\%) and the real-world study by Lee et al. [26] (34.8\%). TACE is a regional therapy combined with targeted chemotherapy and arterial embolization [14], but TACE alone shows poor efficacy. Indeed, the hypoxic state induced by TACE stimulates tumor angiogenesis to bypass the blocked tumor feeding arteries to promote disease progression or metastasis [27, 28]. Incomplete tumor necrosis in the target area could also be involved [29]. Due to the rich blood supply of tumors, complex blood supply arteries, and poor arteriole opening $[19,22,23]$, there are often residual tumor-supporting vessels after TACE.

Regorafenib is a systemic multikinase inhibitor. It can inhibit tumor angiogenesis by inhibiting vascular endothelial growth factor receptor (VEGFR)-1, VEGFR2, VEGFR-3, receptor tyrosine-protein kinase (Tie-2), and other protein kinase activity and play a role in antiangiogenesis [22-24, 26]. In addition, it can also exert multiple antitumor effects by inhibiting multiple kinases involved in tumor proliferation and tumor microenvironment $[22,23]$. Therefore, the combination of TACE and regorafenib can achieve synergistic effects. In addition, although regorafenib and sorafenib have overlapping targets, regorafenib targets a wider range of kinases and has stronger inhibitory effects on VEGFR-2, PDGFR- $\beta$, FGFR-1, and c-Kit. At the same time, regorafenib can also inhibit Tie-2, which has a broader anti-angiogenesis effect $[22,30]$. Therefore, in TACE combined with systemic therapy, regorafenib can be used after progression to sorafenib, with a better prognosis and higher response $[24,26]$.

The factors associated with the PFS, TTP, and OS in the univariable analyses were tumor diameter $>3.75 \mathrm{~cm}$, AFP $>400 \mathrm{ng} / \mathrm{ml}$, dose of regorafenib, and best response to regorafenib. Tumor size is an important staging and prognosis factor of HCC [1]. AFP levels indicate liver damage, and elevated AFP levels have been associated with a poor prognosis of HCC [1,31,32]. The dose of regorafenib was associated with the outcomes, with higher doses achieving better effects. Bruix et al. [24] only used the $160-\mathrm{mg} / \mathrm{d}$ dose, but the actual dose might vary in a real-world setting, and additional studies should examine this. Finally, of course, the patients who achieve $\mathrm{CR}, \mathrm{PR}$, or SD as their best response have a higher likelihood of longer survival and TTP.

There was no grade $5 \mathrm{AE}$ in this study. The complications of TACE are well-known [15, 19, 28, 29], and no novel safety signal was observed. The incidence of grade 3-4 adverse events was $15.8 \%$, much lower than in the RESORCE trial (67\%) [24]. This lower frequency of AEs could be due to a reporting bias and a lack of active surveillance.

We agree that locoregional therapy in patients with metastatic disease is not necessarily the standard of care in cancer in general. Still, the literature contains several patients in whom treating the primary disease led to a better prognosis despite distant metastasis [33-37]. Indeed, death due to metastasis occurs when the affected organs cease their normal functions. Still, in HCC, liver 
dysfunction or gastrointestinal hemorrhage leading to death might occur before the dysfunction of other organs if the liver disease is left untreated [33, 38-44]. Therefore, managing the primary disease might improve the prognosis of these patients [33-37]. We are the first to agree that this approach can be appropriate only in selected patients, but we need data to determine who these patients are. Sharing these data is the prerequisite for science advancement in the field of HCC management.

There was no control group, which is a limitation of the study. Still, the present study provides data about TACE with regorafenib, which could be used as a basis for future trials. Yoo et al. [45] observed no differences among different doses of regorafenib on prognosis, while the present study observed a better prognosis with higher doses. It was a real-world study, not a randomized one, and hence the conclusions must be taken with caution because of the risk of bias for uncontrolled parameters. The initial dose was chosen according to the patients' weight, Child-Pugh grade, and ECOG performance status.

\section{Conclusions}

This retrospective study is limited by its small sample size and short follow-up time, making it difficult to analyze the long-term efficacy outcomes. Despite its retrospective nature, the present study provides real-world evidence indicating that regorafenib combined with TACE was beneficial and tolerable in patients with unresectable HCC. Additional prospective large-scale studies are required for confirmation.

\section{Abbreviations}

TACE: Transarterial chemoembolization; HCC: Hepatocellular carcinoma; OS: Overall survival; TTP: Time to progression; PFS: Progression-free survival; AEs: Adverse events; DCR: Disease control rate; ECOG: Eastern Cooperative Oncology Group; PS: Performance status; BCLC: Barcelona clinic liver cancer; BMI: Body mass index; AFP: a-Fetoprotein; CR: Complete response; PR: Partial response; PD: Progressive disease; SD: Stable disease.

\section{Supplementary Information}

The online version contains supplementary material available at https://doi. org/10.1186/s12876-021-01967-3.

Additional file 1: Table S1. Univariable and multivariable analysis of progression-free survival. Table S2. Univariable and multivariable analysis of time-to-progression. Table S3. Univariable and multivariable analysis of overall survival.

\section{Acknowledgements}

We thank the Scientific Publications Team at Asan Medical Center for the editorial assistance in preparing this manuscript. None of them received compensation for their work.

\section{Authors' contributions}

YH, GC, BS, and JW substantially contributed to study design and critically revised the manuscript for important intellectual content. YH, GC, BS, JW, DY, $H X, Q S, Z L, W Z, L X, B L$, and YZ contributed to data acquisition, analysis, or interpretation, and they all drafted the manuscript. All authors read and gave their final approval to this manuscript.

\section{Funding}

Not applicable.

\section{Availability of data and materials}

The datasets used and/or analyzed during the current study are available from the corresponding author on reasonable request.

\section{Declarations}

\section{Ethics approval and consent to participate}

All experiments were performed in accordance with the Declaration of Helsinki. This study was approved by Chinese Ethics Committee of Registering Clinical Trials (ChiECRCT20210138). The requirement for individual informed consent was waived by Chinese Ethics Committee of Registering Clinical Trials.

\section{Consent for publication}

Not applicable.

\section{Competing interests}

The authors declare that they have no competing interests.

\section{Author details}

${ }^{1}$ Department of Interventional Therapy, National Cancer Center, National Clinical Research Center for Cancer, Cancer Hospital, Chinese Academy of Medical Sciences and Peking Union Medical College, Beijing 100021, China. ${ }^{2}$ Key Laboratory of Carcinogenesis and Translational Research (Ministry of Education/Beijing), Department of Interventional Therapy, Peking University Cancer Hospital and Institute, Beijing 100142, China. ${ }^{3}$ Center of Interventional Oncology and Liver Diseases, Beijing Youan Hospital, Capital Medical University, Beijing 100069, China. ${ }^{4}$ Department of Interventional Radiology and Vascular Surgery, First Hospital, Peking University, Beijing 100034, China.

Received: 5 February 2021 Accepted: 7 October 2021

Published online: 20 October 2021

\section{References}

1. NCCN Clinical Practice Guidelines in Oncology (NCCN Guidelines). Hepatobiliary Cancers. Version 5.2020. Fort Washington: National Comprehensive Cancer Network; 2020.

2. Villanueva A. Hepatocellular carcinoma. N Engl J Med. 2019;380:1450-62.

3. Bray F, Ferlay J, Soerjomataram I, Siegel RL, Torre LA, Jemal A. Global cancer statistics 2018: GLOBOCAN estimates of incidence and mortality worldwide for 36 cancers in 185 countries. CA Cancer J Clin. 2018;68:394-424.

4. Wu M, Tang Z, Liu Y, Chen X, Wang X, Sun Y, et al. Guidelines for diagnosis and treatment of primary liver cancer. Zhongguo Shiyong Waike Zazhi. 2019;2020:121-38.

5. Marrero JA, Kulik LM, Sirlin CB, Zhu AX, Finn RS, Abecassis MM, et al. Diagnosis, staging, and management of hepatocellular carcinoma: 2018 practice guidance by the American Association for the Study of Liver Diseases. Hepatology. 2018;68:723-50.

6. Vogel A, Cervantes A, Chau I, Daniele B, Llovet JM, Meyer T, et al. Hepatocellular carcinoma: ESMO Clinical Practice Guidelines for diagnosis, treatment and follow-up. Ann Oncol. 2018;29:iv238-iv55.

7. Torre LA, Bray F, Siegel RL, Ferlay J, Lortet-Tieulent J, Jemal A. Global cancer statistics, 2012. CA Cancer J Clin. 2015;65:87-108.

8. Chen W, Zheng R, Baade PD, Zhang S, Zeng H, Bray F, et al. Cancer statistics in China, 2015. CA Cancer J Clin. 2016;66:115-32.

9. Jiang JF, Lao YC, Yuan BH, Yin J, Liu X, Chen L, et al. Treatment of hepatocellular carcinoma with portal vein tumor thrombus: advances and challenges. Oncotarget. 2017;8:33911-21. 
10. Hyun MH, Lee YS, Kim JH, Lee CU, Jung YK, Seo YS, et al. Hepatic resection compared to chemoembolization in intermediate- to advanced-stage hepatocellular carcinoma: a meta-analysis of high-quality studies. Hepatology. 2018;68:977-93.

11. Forner A, Reig M, Bruix J. Hepatocellular carcinoma. Lancet. 2018;391:1301-14.

12. Oncology GWCoCSoC. Chinese Society of Clinical Oncology (CSCO) Guidelines for the Diagnosis and Treatment of primary Liver Cancer (2018.V1): People's Medical Publishing House; 2018.

13. Branch CMDAID. Guidelines for the Clinical Practice of Transcatheter Arterial chemoembolization (TACE) for hepatocellular carcinoma in China. Zhonghua Ganzangbing Zazhi. 2019;27:172-81.

14. Jansen MC, van Hillegersberg R, Chamuleau RA, van Delden OM, Gouma DJ, van Gulik TM. Outcome of regional and local ablative therapies for hepatocellular carcinoma: a collective review. Eur J Surg Oncol. 2005;31:331-47.

15. Zhang L, Hu P, Chen X, Bie P. Transarterial chemoembolization (TACE) plus sorafenib versus TACE for intermediate or advanced stage hepatocellular carcinoma: a meta-analysis. PLoS ONE. 2014;9:e100305.

16. Cheng AL, Kang YK, Chen Z, Tsao CJ, Qin S, Kim JS, et al. Efficacy and safety of sorafenib in patients in the Asia-Pacific region with advanced hepatocellular carcinoma: a phase III randomised, double-blind, placebocontrolled trial. Lancet Oncol. 2009;10:25-34.

17. Llovet JM, Ricci S, Mazzaferro V, Hilgard P, Gane E, Blanc JF, et al. Sorafenib in advanced hepatocellular carcinoma. N Engl J Med. 2008;359:378-90.

18. Kudo M, Finn RS, Qin S, Han KH, Ikeda K, Piscaglia F, et al. Lenvatinib versus sorafenib in first-line treatment of patients with unresectable hepatocellular carcinoma: a randomised phase 3 non-inferiority trial. Lancet. 2018;391:1163-73.

19. Kudo M, Ueshima K, Ikeda M, Torimura T, Tanabe N, Aikata H, et al. Randomised, multicentre prospective trial of transarterial chemoembolisation (TACE) plus sorafenib as compared with TACE alone in patients with hepatocellular carcinoma: TACTICS trial. Gut. 2020;69:1492-501.

20. Wu FX, Chen J, Bai T, Zhu SL, Yang TB, Qi LN, et al. The safety and efficacy of transarterial chemoembolization combined with sorafenib and sorafenib mono-therapy in patients with BCLC stage B/C hepatocellular carcinoma. BMC Cancer. 2017;17:645.

21. Choi GH, Shim JH, Kim MJ, Ryu MH, Ryoo BY, Kang YK, et al. Sorafenib alone versus sorafenib combined with transarterial chemoembolization for advanced-stage hepatocellular carcinoma: results of propensity score analyses. Radiology. 2013;269:603-11.

22. Wilhelm SM, Dumas J, Adnane L, Lynch M, Carter CA, Schütz G, et al. Regorafenib (BAY 73-4506): a new oral multikinase inhibitor of angiogenic, stromal and oncogenic receptor tyrosine kinases with potent preclinical antitumor activity. Int J Cancer. 2011;129:245-55.

23. Abou-Elkacem L, Arns S, Brix G, Gremse F, Zopf D, Kiessling F, et al. Regorafenib inhibits growth, angiogenesis, and metastasis in a highly aggressive, orthotopic colon cancer model. Mol Cancer Ther. 2013;12:1322-31.

24. Bruix J, Qin S, Merle P, Granito A, Huang YH, Bodoky G, et al. Regorafenib for patients with hepatocellular carcinoma who progressed on sorafenib treatment (RESORCE): a randomised, double-blind, placebo-controlled, phase 3 trial. Lancet. 2017:389:56-66.

25. PawlikTM, Reyes DK, Cosgrove D, Kamel IR, Bhagat N, Geschwind JF. Phase II trial of sorafenib combined with concurrent transarterial chemoembolization with drug-eluting beads for hepatocellular carcinoma. J Clin Oncol. 2011;29:3960-7.

26. Lee MJ, Chang SW, Kim JH, Lee YS, Cho SB, Seo YS, et al. Real-world systemic sequential therapy with sorafenib and regorafenib for advanced hepatocellular carcinoma: a multicenter retrospective study in Korea. Invest New Drugs. 2020

27. Knox JJ, Cleary SP, Dawson LA. Localized and systemic approaches to treating hepatocellular carcinoma. J Clin Oncol. 2015;33:1835-44.

28. Sergio A, Cristofori C, Cardin R, Pivetta G, Ragazzi R, Baldan A, et al. Transcatheter arterial chemoembolization (TACE) in hepatocellular carcinoma (HCC): the role of angiogenesis and invasiveness. Am J Gastroenterol. 2008;103:914-21.
29. Liu K, Min XL, Peng J, Yang K, Yang L, Zhang XM. The changes of HIF-1a and VEGF expression after TACE in patients with hepatocellular carcinoma. J Clin Med Res. 2016;8:297-302.

30. Wilhelm SM, Carter C, Tang L, Wilkie D, McNabola A, Rong H, et al. BAY 43-9006 exhibits broad spectrum oral antitumor activity and targets the RAF/MEK/ERK pathway and receptor tyrosine kinases involved in tumor progression and angiogenesis. Cancer Res. 2004;64:7099-109.

31. Bai DS, Zhang C, Chen P, Jin SJ, Jiang GQ. The prognostic correlation of AFP level at diagnosis with pathological grade, progression, and survival of patients with hepatocellular carcinoma. Sci Rep. 2017;7:12870.

32. Chan MY, She WH, Dai WC, Tsang SHY, Chok KSH, Chan ACY, et al. Prognostic value of preoperative alpha-fetoprotein (AFP) level in patients receiving curative hepatectomy- an analysis of 1,182 patients in Hong Kong. Transl Gastroenterol Hepatol. 2019;4:52.

33. Uchino K, Tateishi R, Shiina S, Kanda M, Masuzaki R, Kondo Y, et al. Hepatocellular carcinoma with extrahepatic metastasis: clinical features and prognostic factors. Cancer. 2011;117:4475-83.

34. Sinn DH, Cho JY, Gwak GY, Paik YH, Choi MS, Lee JH, et al. Different survival of Barcelona clinic liver cancer stage $C$ hepatocellular carcinoma patients by the extent of portal vein invasion and the type of extrahepatic spread. PLoS ONE. 2015;10:e0124434.

35. Kim KM, Sinn DH, Jung SH, Gwak GY, Paik YH, Choi MS, et al. The recommended treatment algorithms of the BCLC and HKLC staging systems: does following these always improve survival rates for HCC patients? Liver Int. 2016;36:1490-7.

36. Yoo DJ, Kim KM, Jin YJ, Shim JH, Ko GY, Yoon HK, et al. Clinical outcome of 251 patients with extrahepatic metastasis at initial diagnosis of hepatocellular carcinoma: does transarterial chemoembolization improve survival in these patients? J Gastroenterol Hepatol. 2011;26:145-54.

37. Bettinger D, Spode R, Glaser N, Buettner N, Boettler T, Neumann-Haefelin $C$, et al. Survival benefit of transarterial chemoembolization in patients with metastatic hepatocellular carcinoma: a single center experience. BMC Gastroenterol. 2017;17:98.

38. Schutte K, Schinner R, Fabritius MP, Moller M, Kuhl C, lezzi R, et al. Impact of extrahepatic metastases on overall survival in patients with advanced liver dominant hepatocellular carcinoma: a subanalysis of the SORAMIC trial. Liver Cancer. 2020;9:771-86.

39. Reig M, Rimola J, Torres F, Darnell A, Rodriguez-Lope C, Forner A, et al. Postprogression survival of patients with advanced hepatocellular carcinoma: rationale for second-line trial design. Hepatology. 2013;58:2023-31.

40. Iavarone M, Cabibbo G, Biolato M, Della Corte C, Maida M, Barbara M, et al. Predictors of survival in patients with advanced hepatocellular carcinoma who permanently discontinued sorafenib. Hepatology. 2015;62:784-91.

41. Jung SM, Jang JW, You CR, Yoo SH, Kwon JH, Bae SH, et al. Role of intrahepatic tumor control in the prognosis of patients with hepatocellular carcinoma and extrahepatic metastases. J Gastroenterol Hepatol. 2012;27:684-9.

42. Sarpel U, Spivack JH, Berger Y, Heskel M, Aycart SN, Sweeney R, et al. The effect of locoregional therapies in patients with advanced hepatocellular carcinoma treated with sorafenib. HPB (Oxford). 2016;18:411-8.

43. Uka K, Aikata H, Takaki S, Shirakawa H, Jeong SC, Yamashina K, et al. Clinical features and prognosis of patients with extrahepatic metastases from hepatocellular carcinoma. World J Gastroenterol. 2007;13:414-20.

44. Lee HS. Management of patients with hepatocellular carcinoma and extrahepatic metastasis. Dig Dis. 2011;29:333-8.

45. Yoo C, Byeon S, Bang Y, Cheon J, Kim JW, Kim JH, et al. Regorafenib in previously treated advanced hepatocellular carcinoma: Impact of prior immunotherapy and adverse events. Liver Int. 2020;40:2263-71.

\section{Publisher's Note}

Springer Nature remains neutral with regard to jurisdictional claims in published maps and institutional affiliations. 